\title{
Model atmospheres of X-ray bursting neutron stars
}

\author{
V. Suleimanov ${ }^{*, \dagger}$, J. Poutanen**, M. Revnivtsev ${ }^{\ddagger}$ and K. Werner* \\ * Insitute for Astronomy and Astrophysics, Kepler Center for Astro and Particle Physics, Eberhard \\ Karls University, Sand 1, 72076 Tübingen, Germany \\ ${ }^{\dagger}$ Kazan Federal University, Kremlevskaya str. 18, 42008 Kazan, Russia \\ ${ }^{* *}$ Astronomy Division, PO Box 3000, FIN-90014 University of Oulu, Finland \\ ${ }^{*}$ Space Research Institute, Profsoyuznaya str. 84/32, 117997 Moscow, Russia
}

\begin{abstract}
We present an extended set of model atmospheres and emergent spectra of X-ray bursting neutron stars in low mass X-ray binaries. Compton scattering is taken into account. The models were computed in LTE approximation for six different chemical compositions: pure hydrogen and pure helium atmospheres, and atmospheres with a solar mix of hydrogen and helium and various heavy elements abundances; $Z=1,0.3,0.1$, and $0.01 Z_{\odot}$, for three values of gravity, $\log g=14.0,14.3$, and 14.6 and for 20 values of relative luminosity $l=L / L_{\mathrm{Edd}}$ in the range $0.001-0.98$. The emergent spectra of all models are fitted by diluted blackbody spectra in the observed RXTE/PCA band $3-20$ $\mathrm{keV}$ and the corresponding values of color correction factors $f_{\mathrm{c}}$ are presented. We also show how to use these dependencies to estimate the neutron star's basic parameters.
\end{abstract}

Keywords: radiative transfer - stars: neutron - X-rays: bursts - X-rays: individual: 4U 1724-307 PACS: 97.10.Ex,97.10.Nf,97.10.Pg,97.60.Jd,97.80.Jp

The most important and useful sources for the aim of neutron stars (NSs) $M$ and $R$ finding are X-ray bursting NSs with photospheric radius expansion [1]. The relation between observed normalization $K$ (for blackbody fit of spectra) and the real ratio of NS radius $R$ to distance on late outburst phases is:

$$
K^{1 / 2}=\frac{R_{\mathrm{BB}}(\mathrm{km})}{D_{10}}=\frac{R(\mathrm{~km})}{f_{\mathrm{c}}^{2} D_{10}}(1+z),
$$

where $D_{10}$ is the distance in units of $10 \mathrm{kpc}$, and $f_{\mathrm{c}}=T_{\mathrm{c}} / T_{\mathrm{eff}}$ is a color correction factor. Therefore, on these phases $K(t)$ dependence reflects $f_{\mathrm{c}}(t)$ dependence only. We suggest to fit the observed $K^{-1 / 4}-F$ relation by a theoretical $f_{\mathrm{c}}-l \equiv L / L_{\text {Edd }}$ relation, where $F$ is the integral observed flux. From this fit we can obtain two independent values: $R(\mathrm{~km}) \times(1+z) / D_{10}$ and $F_{\mathrm{Edd}} \sim L_{\mathrm{Edd}} /\left((1+z) D_{10}^{2}\right)$. Combining these values, we can obtain an observed $M / R$ relation, which is independent on the distance and physically corresponds to a maximum possible effective temperature on the NS surface. If the distance is known we can find $M$ and $R$ simultaneously. For this method extended theoretical $f_{\mathrm{c}}(l)$ calculations are necessary.

We computed model atmospheres of X-ray bursting NSs subject to the constraints of hydrostatic and radiative equilibrium assuming planar geometry in LTE approximation with Compton scattering taken into account (see details of the code in $[2,3]$ ).

We calculated an extended set of NS model atmospheres with 6 chemical compositions (pure $\mathrm{H}, \mathrm{He}$, and solar $\mathrm{H} / \mathrm{He}$ mixture with $Z=1,0.3,0.1$ and $0.01 Z_{\odot}$ ), 3 sur- 

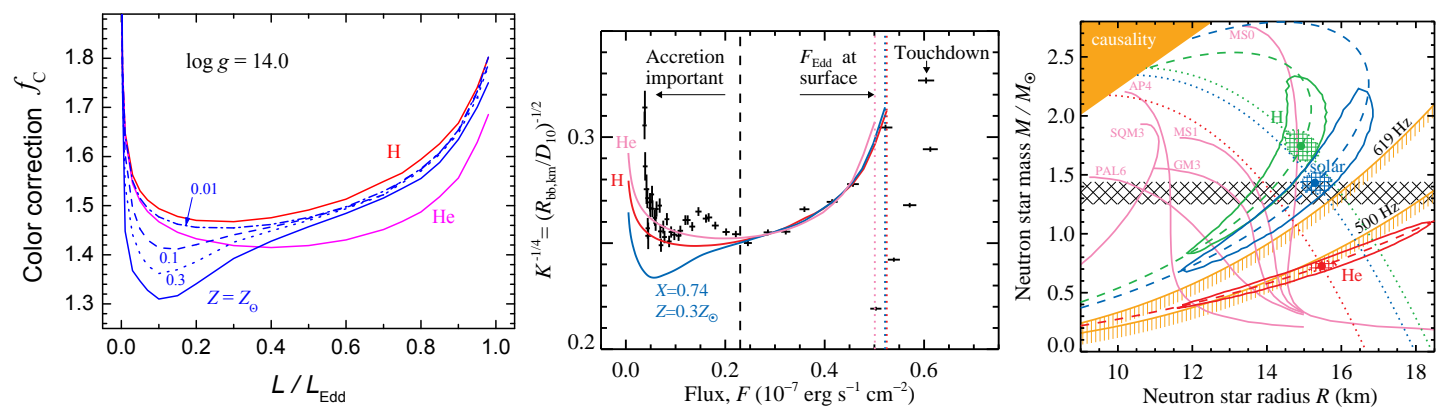

FIGURE 1. Left: Dependence of color correction factors on the relative luminosity for low gravity and various chemical compositions in NS atmosphere models. Middle: Comparison of the observed dependence of $K^{-1 / 4}-F$ for $4 \mathrm{U} 1724-307$ (croses) to the best fit theoretical models $f_{\mathrm{c}}-l$. Right: Constraints on mass and radius of the neutron star 4U 1724-307.

face gravities: $\log g=14.0,14.3$ and 14.6, and 20 luminosities $L: 0.001,0.003,0.01$, $0.03,0.05,0.07,0.1,0.15,0.2,0.3,0.4,0.5,0.6,0.7,0.75,0.8,0.85,0.9,0.95$, and $0.98 L_{\text {Edd }}$. Corresponding $T_{\text {eff }}$ were calculated from $L$ using $\log g$ and chemical composition. The model emergent redshifted spectra were fitted by diluted blackbody spectra $F_{\mathrm{E}}=w B_{\mathrm{E}}\left(f_{\mathrm{c}} T_{\mathrm{eff}}\right)$ in the $R X T E /$ PCA energy band $3-20 \mathrm{keV}$. Here $w \approx f_{\mathrm{c}}^{-4}$ is the dilution factor. The accepted redshifts were calculated from $\log g$ assuming $M=1.4 M_{\odot}$. Results are partially presented in Fig. 1, left panel.

We fitted the observed $K^{-1 / 4}-F$ relation, obtained for the extremely long outburst of 4U 1724-307 in November 8, 1996 (RXTE, [4]) by computed $f_{\mathrm{c}}-l$ relations. We obtained limitations on $R$ and $M$ for the adopted distance $D=5.3 \pm 0.6 \mathrm{kpc}$ [5] and various chemical compositions, see Fig. 1 . The values of $M$ and $R$ obtained for $\mathrm{H}-$ rich atmospheres correspond to a stiff Equation of State in the inner NS core. Helium atmospheres are not acceptable. More details can be found in [6].

\section{ACKNOWLEDGMENTS}

The work is supported by the DFG grant SFB / Transregio 7 "Gravitational Wave Astronomy" (V.S.), Russian Foundation for Basic Research (grant 09-02-97013-p-povolzhe-a, V.S.), and the Academy of Finland (grant 127512, J.P.).

\section{REFERENCES}

1. W. H. G. Lewin, J. van Paradijs, and R. E. Taam, Space Sci. Rev. 62, 223-389 (1993).

2. V. Suleimanov, and J. Poutanen, MNRAS 369, 2036-2048 (2006).

3. V. Suleimanov, and K. Werner, Astron. and Astrophys. 466, 661-666 (2007).

4. S. V. Molkov, S. A. Grebenev, and A. A. Lutovinov, Astron. and Astrophys. 357, L41-L44 (2000).

5. S. Ortolani, E. Bica, and B. Barbuy, Astron. and Astrophys. 326, 614-619 (1997).

6. V. Suleimanov, J. Poutanen, M. Revnivtsev, and K. Werner, arXiv:1004.4871 (2010). 